\title{
ESTUDIO MORFO-ANATÓMICO COMPARADO EN PECÍOLOS DE LAS ESPECIES DE AspleNIUM (AspleniaceAe) DE Argentina
}

\author{
Comparative morpho-AnATOMICAL STUdy in PETIOLES OF AsplenIUM \\ (Aspleniaceae) SPECIES from Argentina
}

\author{
María A. Ganem ${ }^{1 *} \mathbb{D}$, María L. Luna ${ }^{2,3}$ (D) Osvaldo Ahumada ${ }^{1}$ y Gabriela E. \\ Giudice ${ }^{2}$ iD
}

\begin{abstract}
1. Cátedra de Botánica General, Facultad de Ciencias Agrarias, Universidad Nacional de Jujuy, Alberdi $n^{\circ} 47,(4600)$ San Salvador de Jujuy, Argentina.

2. Cátedra Morfología Vegetal, Facultad de Ciencias Naturales y Museo, Universidad Nacional de La Plata. Edificio Anexo Museo, Boulevard 120 entre 61 y 64, (1900) La Plata, Argentina. 3. Comisión de Investigaciones Científicas de la Provincia de Buenos Aires, Camino Gral. Belgrano y 526, (1900) La Plata, Argentina.
\end{abstract}

*aleganem@hotmail.com

\section{Citar este artículo}

GANEM, M. A., M. L. LUNA, O. AHUMADA \& G. E. GIUDICE. 2019. Estudio morfo-anatómico comparado en pecíolos de las especies de Asplenium (Aspleniaceae) de Argentina. Bol. Soc. Argent. Bot. 54: 191-201.

DOI: http://dx.doi. org/10.31055/1851.2372.v54. n2.24364

Recibido: 28 Septiembre 2018

Aceptado: 9 febrero 2019

Publicado: 30 Junio 2019

Editora: Ana María Gonzalez

ISSN versión impresa 0373-580X

ISSN versión on-line 1851-2372

\section{SUMMARY}

Background and aims: The morphology and the anatomy of the petioles provide useful information for taxonomic issues in ferns. The aim of this study was to analyze the diagnostic characters of petioles in 37 species of Asplenium that grow in Argentina, to contribute to their identification and to ascertain on their adaptive significance.

M\&M: Herbarium specimens and fresh material were employed. Materials were analyzed under stereoscopic and light microscopes; in the latter case, the samples were processed following the conventional histological techniques. The characters analyzed were: colour, length, presence of wings, sub-epidermic mechanical tissue, aeration lines, circumendodermal band (CB), shape and number of vascular bundles and xylem contour.

Results: Some characteristics of the petioles allow the grouping of species. The diagnostic features are: colour, presence/absence of wings, number of vascular bundles, bundles contour, xylem contour and presence/absence of $\mathrm{CB}$.

Conclusions: Petiole characters alone do not allow the identification of the taxa at specific level, although along with others they contribute to the systematics of the genus. The anatomy of the petiole does not show in general terms relationship with the habit of these plants. The type and distribution of subepidermic mechanical tissue and the presence of CB are associated with species that inhabit exposed and dry environments, but these features are not exclusive of the taxa that grow under these conditions.

\section{KEY WORDS}

Asplenium, circumendodermal band, petiole, taxonomy, vascular bundles.

\section{RESUMEN}

Introducción y objetivos: Las características externas e internas de los pecíolos brindan información útil para los estudios taxonómicos en helechos. El objetivo de este trabajo fue analizar los caracteres diagnósticos del pecíolo en las 37 especies de Asplenium que crecen en Argentina, para contribuir a su identificación e indagar sobre su significado adaptativo.

M\&M: Se emplearon especímenes de herbario y material fresco. Los materiales se analizaron con microscopios estereoscópico y óptico; en el último caso las muestras fueron procesadas siguiendo las técnicas histológicas convencionales. Los caracteres analizados fueron: color, longitud, presencia de alas, tejido mecánico sub-epidérmico, líneas de aireación, banda circumendodermal (BC), forma y número de los haces vasculares y contorno del xilema.

Resultados: Algunas características de los pecíolos permiten el agrupamiento de especies. Los caracteres diagnósticos son: color, presencia/ausencia de alas, número de haces vasculares, contorno de los haces, contorno del xilema y presencia/ausencia de BC.

Conclusiones: Los caracteres del pecíolo por si solos no permiten la identificación de los taxa a nivel específico, si bien junto con otros contribuyen a la sistemática del género. La anatomía del pecíolo no evidencia en general relación con el hábito de estas plantas. El tipo y la disposición del tejido mecánico sub-epidérmico y la presencia de $\mathrm{BC}$ se asocian a especies que crecen en ambientes expuestos y secos, pero no son exclusivos de los taxa que crecen bajo estas condiciones.

\section{Palabras clave}

Asplenium, banda circumendodermal, haces vasculares, pecíolo, taxonomía. 


\section{INTRODUCCIÓN}

El género Asplenium (Aspleniaceae) se halla representado en Argentina por 37 especies distribuidas principalmente en el Noroeste, Noreste y Centro, y unas pocas en el área Patagónica (Ponce, 1996; Giudice et al. 2013; Ganem et al. 2016).

Las Aspleniaceae son de interés para los estudios morfológicos, dado su amplio rango de distribución y el elevado número de especies que contemplan (más de 700, Schneider 1997). Ello resulta en una gran variedad de caracteres morfológicos sistemáticos y adaptativos, asociados al hábito terrestre, saxícola o epífito.

El género Asplenium comprende plantas con rizomas erectos a cortamente rastreros, con escamas clatradas; pecíolos herbáceos a lignificados, acanalados dorsalmente, con o sin alas; láminas enteras o divididas (1-3 pinnadas), herbáceas a subcarnosas, glabras, a veces con escamas en los raquis, venas libres o raramente anastomosadas; soros oblongos a lineares con indusio de inserción lateral, de margen entero a eroso, a veces ausente; esporas monoletes, castaño-oscuras, con perisporio plegado a crestado con diferentes ornamentaciones; número básico de cromosomas $\mathrm{x}=36$ (Smith, 1981; Tryon \& Stolze, 1993; Ganem et al., 2016).

En las Aspleniaceae las características anatómicas de las raíces junto con la morfología de los rizomas, de las láminas y de las esporas aportan información diagnóstica (Giudice et al. 2002, 2013; Ganem et al. 2007, 2014, 2016; Wetzel et al. 2017). En cuanto a los pecíolos, su morfología y anatomía han probado brindar caracteres sistemáticos diagnósticos, como el color y el número de surcos (1 a 3 ) generalmente en la cara adaxial, la presencia de alas, así como el número de haces y el contorno del xilema (Keating, 1968; Kato, 1972; Ogura, 1972; Lin \& De Vol, 1977 a, b; Umikalsom, 1992; Gabancho et al. 2011). Asimismo, la presencia o ausencia de alas en los pecíolos es un carácter diagnóstico a nivel de especie en Asplenium (Lin \& De Vol, 1977a).

El objetivo de este trabajo fue realizar el análisis comparado de los caracteres morfo-anatómicos de los pecíolos, con el fin de aportar información a la sistemática del género, especialmente de los taxones que crecen en Argentina, e indagar sobre su relación con los hábitos y los distintos ambientes donde crecen estas plantas.

\section{Materiales y MÉTodos}

El material analizado provino principalmente de ejemplares de herbario (ca. de 400 ejemplares) y de material recolectado en el campo. Los ejemplares de referencia fueron depositados en los herbarios LP y JUA.

Para los estudios con microscopio óptico (MO) se utilizaron como mínimo 3 individuos por especie. Las muestras fueron procesadas siguiendo técnicas convencionales (D’ Ambroggio de Argüeso, 1986; Zarlavsky, 2014), las que se describen brevemente a continuación.

Para recuperar el material de herbario, porciones de pecíolo fueron colocadas en vasos de precipitado con agua y unas gotas de detergente no iónico, y calentadas suavemente. En el caso del material fresco, este se fijó en formol- alcohol etílico- ácido acético (FAA) hasta su procesamiento.

Se realizaron cortes en las porciones basal y media de los pecíolos, tanto a mano alzada con cuchillas descartables como con micrótomo de congelación (criótomo). Las secciones obtenidas fueron coloreadas con safranina y montadas con gelatina-glicerina.

El material fue observado y fotografiado con microscopio estereoscópico Nikon SMZ 1000 y microscopio óptico Nikon E200.

\section{Material de referencia}

Asplenium abscissum Willd. Prov. Misiones: Dpto. Cainguas, Arroyo del Valle, Reserva UNLP, sobre arroyo Tateto, 10-I-2003, Márquez et al. 46 (LP).

A. achalense Hieron. Prov. Tucumán: Dpto. Monteros, Quebrada Pueblo Viejo, epífito, 1-I1965, de la Sota 4060 (LP).

A. alatum Humb. \& Bonpl. ex Wild. Prov. Misiones: Dpto. San Pedro, terrestre, creciendo en grietas entre rocas - recubriendo salpicaduras provenientes del salto, 10-I-1991, Johnson 0153 (SI).

A. argentinum Hieron. Prov. Misiones: Dpto. Guaranii: en selva marginal, curso de arroyo, hierba saxícola de color verde, 23-IX-1993, Rodriguez et al. 00732 (LP).

A. auriculatum Sw. Prov. Misiones: 19-VIII1972, Eskuche 01760 (SI).

A. auritum Sw. Prov. Jujuy: Dpto. Libertador, Gral. San Martín, Parque Nacional Calilegua, 25V-2011, Ganem et al. 58 (JUA). 
A. balansae (Baker) Sylvestre. Prov. Misiones: Dpto. Cainguás, Reserva Privada del Arroyo Cuña Pirú, 3-I-2005, Márquez \& Biganzoli F. 701 (SI).

A. brasiliense Sw. Prov. Misiones: Dpto. San Pedro, Reserva de Biósfera Yabotí, Parque Provincial Esmeralda, sendero junto al arroyito de la "toma de agua", 22-XI-2006, Belgrano et al. 583 (SI).

A. claussenii Hieron. Prov. Jujuy: Dpto. Manuel Belgrano, Parque Botánico Los Perales, 10-XI1994, Ganem 06 (JUA). Dpto. Palpalá, sierras de Zapla, 4-VI-2014, Ganem et al. 295 (JUA).

A. cuspidatum Lam. Prov. Jujuy: Dpto. Ledesma, Parque Nacional Calilegua, 31-V-2016, Ganem et al. 301 (JUA).

A. dareoides Desv. Prov. Tierra del Fuego: Río Cambaceres, 17-III-1967, Goodall 766 (LP).

A. depauperatum Fée. Prov. Tucumán: Dpto. Graneros, 9-V-1964, Garenzo 2115 (LIL).

A. formosum Willd. Prov. Salta: Dpto. Anta, Parque Nacional El Rey, 14-VI-1980, A. Brown 1038 (LP).

A. gastonis Fée. Prov. Misiones: Dpto. San Pedro, Reserva Esmeralda, laguna semipermanente a la izq. del camino que va al $\mathrm{A}^{\circ}$ Florida, 26-IV-2007, Márquez et al. 209 (CTES).

A. gilliesi Hook. Prov. Jujuy: Dpto Valle Grande, Co. Hermoso, faldeo oeste, común en microambientes muy húmedos $\mathrm{y}$ protegidos (al abrigo de rocas) 3390 msnm, 21-I-1995, Ayarde 477 (LIL).

A. harpeodes Kunze. Prov. Jujuy: Dpto. Ledesma, Parque Nacional Calilegua, Aguada del tigre, 5-VI-2014, Ganem et al. 300 (JUA).

A. inaequilaterale Willd. Prov. Misiones: Dpto. San Pedro, Parque Provincial Moconá, 7-V-1999, Deginiani et al. 1085 (SI).

A. kunzeanum Klotzsch ex Rosenst. Prov. Misiones: Dpto San Pedro, R. P. Caa Yari, 2709'S 53⒌'W, 360 msnm, 12-III-2009, Marquez et al. 249 (LP).

A. lilloanum de la Sota. Prov. Jujuy: Dpto. Sta. Bárbara, del Fuerte a Cachipunco, 20-II-1985, Kiesling et al. 5456 (SI).

A. lorentzii Hieron. Prov. Jujuy: Dpto. Manuel Belgrano, Yala, 3-VI-2014, Ganem et al. 298 (JUA).

A. monanthes L. Prov. Jujuy, Dpto. Manuel Belgrano, Tiraxi, 5-VI-2014, Ganem et al. 290 (JUA).
A. mucronatum C. Presl Prov. Misiones: Dpto. Iguazú, Puerto Península, 19-VIII-1972, selva semi explotada, epifíto, Eskuche 01761 (SI).

A. palmeri Maxon. Prov. Córdoba: Dpto. Ascochinga, IX-1936, Nicora 972 (SI).

A. papaverifolium (Kunze) Viane. Prov. Santa Cruz: Dpto. Lago Argentino, I-1958, James 1371 (SI).

A. praemorsum Sw. Prov. Salta: Dpto. La Caldera, Camino de cornisa Salta-Jujuy, 4-III-1999, Ganem 124 (JUA).

A. pulchellum Raddi. Prov. Misiones: Dpto. Cainguás, predio UNLP, Reserva Valle del Arroyo Cuña Pirú, alrededores del balneario, borde del arroyo C.P. $27^{\circ} 05^{\prime} 12^{\prime}$ 'S $54^{\circ} 57^{\prime} 11^{\prime \prime} \mathrm{W}$ barranca del arroyo, terrestre, sotobosque, 22-I-2004, Biganzoli et.al.1293 (SI).

A. pumilum Sw. Prov. Salta: Dpto. Capital, Río Mojotoro, margen derecho, unos $1500 \mathrm{~m}$ al este del puente de FCGR, en barranca húmeda, 22-III-1987, C. Palací 917 (LP).

A. resiliens Kunze. Prov. Jujuy: Dpto. Manuel Belgrano, Parque Botánico Los Perales, 10-XI-1994, Ganem 08 (JUA).

A. scandicinum Kaulf. Prov. Misiones: Dpto. Caa Yari, 18-V-2009, Márquez et al. 257 (LP).

A. sellowianum C. Presl ex Hieron. Prov. Buenos Aires: Dpto. Ensenada, Punta Lara, 29-IX-1974, Zardini 355-1 (LP).

A. serra Langsd. \& Fisch. Prov. Jujuy: Dpto. Ledesma, Parque Nacional Calilegua, 24-V-2011, Ganem et al. 272 (JUA).

A. serratum L. Prov. Misiones: Dpto. Gral.Manuel Belgrano, Reserva de Vida Silvestre Urugua-í, sendero al cerro Largo $25^{\circ} 59^{\prime} 85^{\prime} \mathrm{S} 54^{\circ} 06^{\prime} 35^{\prime \prime} \mathrm{W}$ $370 \mathrm{msnm}$, en el sotobosque, 24-IX-2004, Múlgura de Romero et al. 3940 (SI).

A. squamosum L. Prov. Jujuy: Dpto. Manuel Belgrano, Yala, 3-VI-2014, Ganem et al. 299 (JUA).

A. trilobum Cav. Prov. Chubut: Dpto. Cushamen, Parque Nacional Lago Puelo, Arroyo Melo, Vidoz 140 (LP).

A. triphyllum C.Presl. Prov. Jujuy: Dpto. Rinconada, 1-III-1964, H. Schwabe et al. 905 (LP).

A. ulbrichtii Rosenst. Prov. Buenos Aires: Pdo. Ensenada, Punta Lara, arroyo Capitán, 22-X-1985, Castro 99 (LP).

A. uniseriale Raddi. Prov. Misiones: Dpto. San Pedro, Parque Provincial Moconá, picada a la gruta, Marque \& Yañez 51 (LP). 


\section{Resultados}

Los caracteres morfológicos y anatómicos del pecíolo analizados en las 37 especies de Asplenium se muestran en la Tabla 1.

Los pecíolos pueden ser verdosos o castaños a castaño oscuro, lustroso, y disponerse en forma erecta o péndula (Fig. 1A-B). Pueden ser cortos $(1-7 \mathrm{~cm})$, de $1 / 10$ a $1 / 20$ de la longitud de la lámina, o tan largos $(40-50 \mathrm{~cm})$ como la lámina y por lo general tienen un surco dorsal (adaxial).

El indumento consiste en pelos pluricelulares o escamas lanceoladas clatradas semejantes a las del rizoma, o filiformes, generalmente ubicadas en la zona basal (Fig. 1C-E).

Usualmente se observan extensiones laterales flanqueando los pecíolos, denominadas alas, que presentan variabilidad en su desarrollo. Éstas pueden medir 1-2 $\mathrm{mm}$ de ancho como en A. alatum (Fig. 1F), o tener escaso desarrollo (menor a 1 $\mathrm{mm}$ ) como en $A$. abscissum y $A$. argentinum. Las alas pueden extenderse en los raquis, como en $A$. alatum, A. clausenii y $A$. lilloanum. En algunas especies no se observan alas, como en $A$. dareoides y A. uniseriale (Fig. 2A-C).

En sección transversal los pecíolos muestran un contorno redondeado en la mayoría de las especies, ocasionalmente oblongo (Fig. 2B-D). Externamente se distingue la epidermis y a continuación la corteza y los haces vasculares. La epidermis es uniestratificada y por debajo se observa un tejido subepidérmico mecánico, que tiene diferentes características, grado de desarrollo y disposición (Fig. 2A). Éste puede estar constituido por tejido esclerenquimático con distintas capas de espesor (entre 2 a 5) o un parénquima de paredes algo engrosadas (Fig. 2E-F, H-J). Puede consistir en una banda continua (interrumpida a la altura de las alas) o encontrarse ubicado solo hacia la cara abaxial (Fig. 2A).

En varias especies, entre ellas $A$. dareoides, A. praemorsum y $A$. ulbrichtii, se observan en el pecíolo interrupciones del tejido subepidérmico, llamadas líneas continuas laterales de aireación, que cumplen funciones de ventilación (Fig. 2 D-G).

En casi la totalidad de los pecíolos ingresan basalmente dos haces vasculares elípticos o en forma de $\mathrm{C}$, donde el xilema presenta también forma de C, o en algunos casos forma de "hipocampo" dada por la curvatura del protoxilema (por ejemplo en $A$. serra) (Fig. 3A-C, E). Estos haces se unen en la zona media para formar un único haz rodeado de una endodermis, donde el xilema presenta forma de $\mathrm{X}$ o $\mathrm{V}$, raramente de $\mathrm{U}$ (A. cuspidatum) (Fig. 3D, I). En aquellos pecíolos en los que el xilema de los haces presenta en la zona basal forma de hipocampo, esta figura puede apreciarse también cuando los haces se unen en forma de $\mathrm{X}$ o de $\mathrm{V}$ en la zona media (Fig. 3D). En el pecíolo de A. auritum ingresa un solo haz vascular con forma de $\mathrm{U}$, el cual se mantiene a lo largo de todo el recorrido, presentando también el xilema forma de U (Fig. 3F).

Por fuera de la endodermis se distingue generalmente una banda circumendodermal $(\mathrm{BC})$ (Fig. 3G-I). Ésta se caracteriza por sus células de paredes engrosadas en mayor o menor grado, y su coloración castaño oscuro. La BC suele ser continua pero en ocasiones está interrumpida, como en A. serratum (Fig. 3I). En algunos pecíolos no se observa la BC. En estos casos se distinguen grupos de células esclerenquimatosas rodeando el haz vascular (por ejemplo A. auritum) (Fig. 3F).

Tabla 1. Caracteres morfo-anatómicos de los pecíolos y hábito de las especies de Asplenium de Argentina. Alas presentes (p). TMS: tejido mecánico subepidérmico continuo (c), solo abaxial (a). BC: banda circumendodermal (presente: p). GCE: grupos de células de paredes engrosadas. LA: Líneas de aireación (presentes: p). P:L: Relación peciolo/lámina. Número y contorno de los haces vasculares corresponde a la zona basal del peciolo. Hábito: T: terrestre. E: epifita S: saxícola

\begin{tabular}{|c|c|c|c|c|c|c|c|c|c|}
\hline Especie & color & alas & TMS & $\mathrm{BC}$ & LA & $P: L$ & $\begin{array}{c}\text { Número } \\
\text { haces } \\
\text { zona } \\
\text { basal }\end{array}$ & $\begin{array}{c}\text { Contorno } \\
\text { del xilema } \\
\text { zona } \\
\text { media }\end{array}$ & $\begin{array}{l}\text { Hábito } \\
\text { de la } \\
\text { planta }\end{array}$ \\
\hline A. absscisum & verde castaño & $p$ & a & $p$ & 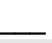 & $1 / 1$ & 2 & $x$ & TSE \\
\hline A. achalense & castaño oscuro & $p$ & $\begin{array}{l}\text { c (interrumpido } \\
\text { en alas) }\end{array}$ & - & $p$ & $1: 2 / 3$ & 2 & $x$ & $E$ \\
\hline
\end{tabular}


Tabla 1. Continuación.

\begin{tabular}{|c|c|c|c|c|c|c|c|c|c|}
\hline Especie & color & alas & TMS & $\mathrm{BC}$ & LA & $P: L$ & $\begin{array}{c}\text { Número } \\
\text { haces } \\
\text { zona } \\
\text { basal }\end{array}$ & $\begin{array}{c}\text { Contorno } \\
\text { del xilema } \\
\text { zona } \\
\text { media }\end{array}$ & $\begin{array}{c}\text { Hábito } \\
\text { de la } \\
\text { planta }\end{array}$ \\
\hline A. alatum & castaño oscuro & $\mathrm{p}$ & $\begin{array}{c}\text { c (interrumpido } \\
\text { en alas) }\end{array}$ & - & $\mathrm{p}$ & $1 / 2$ & 2 & $x$ & ES \\
\hline A. argentinum & castaño oscuro & $\mathrm{p}$ & $\begin{array}{c}\text { c (interrumpido } \\
\text { en alas) }\end{array}$ & - & - & $1 / 3$ & 2 & $x$ & TS \\
\hline A. auriculatum & verde grisáceo & & - & $(\overline{\mathrm{GCE}})$ & - & $1 / 3$ & 2 & V & E S \\
\hline A. auritum & verde & $\mathrm{p}$ & $\begin{array}{c}\text { c (interrumpido } \\
\text { en alas) }\end{array}$ & - & $\mathrm{p}$ & $1 / 1$ & 1 & $U$ & E S \\
\hline A. balansae & castaño & $\longrightarrow$ & a & $(\overline{\mathrm{GCE}})$ & - & $1 / 1$ & 2 & $x$ & $\mathrm{~T}$ \\
\hline A. brasiliense & castaño & $\mathrm{p}$ & - & GCE & - & $1: 6 / 20$ & 2 & $x$ & $\mathrm{~T}$ \\
\hline A. claussenii & castaño & $\mathrm{p}$ & $\begin{array}{c}\text { c (interrumpido } \\
\text { en alas) }\end{array}$ & - & - & $1 / 3$ & 2 & $x$ & $\mathrm{~T}$ \\
\hline A. cuspidatum & castaño & $\mathrm{p}$ & $\begin{array}{l}\text { c (interrumpido } \\
\text { en las alas) }\end{array}$ & GCE & $p$ & $1: 2 / 3$ & 2 & $U$ & E T \\
\hline A. daeroides & castaño oscuro & 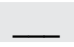 & a & - & $p$ & $1: 4 / 6$ & 2 & $x$ & EST \\
\hline A. depauperatum & castaño oscuro & $\mathrm{p}$ & $\begin{array}{c}\text { c (interrumpido } \\
\text { en las alas) }\end{array}$ & $(\overline{\mathrm{GCE}})$ & - & $1: 10 / 15$ & 2 & $x$ & TS \\
\hline A. formosum & castaño oscuro & $\mathrm{p}$ & $\begin{array}{c}\text { c (interrumpido } \\
\text { en las alas) }\end{array}$ & $\mathrm{p}$ & - & $\begin{array}{c}1 / 4 \\
1 / 10\end{array}$ & 2 & $x$ & ES \\
\hline A. gastonis & verde-castaño & $\mathrm{p}$ & $\begin{array}{c}\text { c (interrumpido } \\
\text { en las alas) }\end{array}$ & GCE & $\mathrm{p}$ & $1 / 2$ & 2 & V & ES \\
\hline A. gilliesii & verde-castaño & 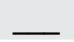 & - & - & - & $1: 4 / 5$ & 2 & $\mathrm{X}$ & $\mathrm{T}$ \\
\hline A. harpeodes & castaño oscuro & $\mathrm{p}$ & $\begin{array}{c}\text { c (interrumpido } \\
\text { en las alas) }\end{array}$ & - & - & $1: 3 / 4$ & 2 & $x$ & E T \\
\hline A. inaequilaterale & verde-castaño & $\mathrm{p}$ & a & $\mathrm{p}$ & $\longrightarrow$ & $\begin{array}{l}1: 2 \text { o } \\
\text { menor }\end{array}$ & 2 & $x$ & $\mathrm{~T}$ \\
\hline A. kunzeanum & verde-castaño & $\mathrm{p}$ & $\begin{array}{l}\text { c (interrumpido } \\
\text { en las alas) }\end{array}$ & $\mathrm{p}$ & - & $1 / 2$ & 2 & $x$ & TE \\
\hline A. lilloanum & castaño oscuro & $\mathrm{p}$ & $\begin{array}{l}\text { c (interrumpido } \\
\text { en las alas) }\end{array}$ & $\mathrm{p}$ & $\longrightarrow$ & $1 / 10$ & 2 & $x$ & $S$ \\
\hline A. lorentzii & verde-castaño & $\mathrm{p}$ & $\begin{array}{c}\text { c (interrumpido } \\
\text { en las alas) }\end{array}$ & - & $\mathrm{p}$ & $1 / 10$ & 2 & $x$ & $S$ \\
\hline A. monanthes & $\begin{array}{l}\text { castaño oscuro } \\
\text { lustroso }\end{array}$ & - & c & $\longrightarrow$ & - & $1 / 5$ & 2 & $x$ & SE \\
\hline A. mucronatum & castaño claro & - & a & - & 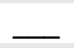 & $1: 10 / 15$ & 2 & $x$ & $\mathrm{E}$ \\
\hline A. palmeri & $\begin{array}{l}\text { castaño oscuro } \\
\text { lustroso }\end{array}$ & $\mathrm{p}$ & $\begin{array}{l}\text { c (interrumpido } \\
\text { en las alas) }\end{array}$ & $x$ & - & $1 / 20$ & 2 & V & $S$ \\
\hline A. papaverifolium & castaño claro & - & c & GCE & - & $1 / 2$ & 2 & $x$ & TS \\
\hline A. praemorsum & $\begin{array}{l}\text { castaño oscuro } \\
\text { lustroso }\end{array}$ & - & c & - & $\mathrm{p}$ & $1: 3 / 4$ & 2 & $x$ & ES \\
\hline A. pulchellum & $\begin{array}{l}\text { verde-castaño } \\
\text { lustroso }\end{array}$ & $\mathrm{p}$ & $\begin{array}{l}\text { c (interrumpido } \\
\text { en las alas) }\end{array}$ & $\mathrm{p}$ & $\mathrm{p}$ & $1 / 7$ & 2 & $x$ & ES \\
\hline A. pumilum & castaño & $\mathrm{p}$ & $\begin{array}{l}\text { c (interrumpido } \\
\text { en las alas) }\end{array}$ & GCE & $\mathrm{p}$ & $1 / 1$ & 2 & V & $\mathrm{T}$ \\
\hline A. resiliens & $\begin{array}{l}\text { castaño oscuro } \\
\text { lustroso }\end{array}$ & $\mathrm{p}$ & $\begin{array}{c}\text { c (interrumpido } \\
\text { en las alas) }\end{array}$ & $\mathrm{p}$ & - & $1 / 6$ & 2 & $x$ & S \\
\hline A. scandicinum & verde-castaño & $\mathrm{p}$ & $\begin{array}{c}\text { c (interrumpido } \\
\text { en las alas) }\end{array}$ & - & - & $1 / 6$ & 2 & $x$ & $\mathrm{E}$ \\
\hline A. sellowianum & castaño & $\mathrm{p}$ & $\begin{array}{c}\text { c (interrumpido } \\
\text { en las alas) }\end{array}$ & $\mathrm{p}$ & $\mathrm{p}$ & $1: 4 / 5$ & 2 & $x$ & TS \\
\hline A. serra & castaño oscuro & $\overline{-}$ & c & $\overline{-}$ & $p$ & $1: 3 / 4$ & 2 & V & TES \\
\hline
\end{tabular}


Bol. Soc. Argent. Bot. 54 (2) 2019

Tabla 1. Continuación.

\begin{tabular}{|c|c|c|c|c|c|c|c|c|c|}
\hline Especie & color & alas & TMS & $\mathrm{BC}$ & LA & $P: L$ & $\begin{array}{c}\text { Número } \\
\text { haces } \\
\text { zona } \\
\text { basal }\end{array}$ & $\begin{array}{c}\text { Contorno } \\
\text { del xilema } \\
\text { zona } \\
\text { media }\end{array}$ & $\begin{array}{c}\text { Hábito } \\
\text { de la } \\
\text { planta }\end{array}$ \\
\hline A. serratum & verde-castaño & & c & $p$ & $p$ & $1 / 10$ & 2 & $x$ & $E$ \\
\hline A. squamosum & verde-castaño & $p$ & $\begin{array}{l}\text { c (interrumpido } \\
\text { en las alas) }\end{array}$ & - & $p$ & $1 / 2$ & 2 & $\mathrm{X}$ & $\mathrm{T}$ \\
\hline A. trilobum & verde & 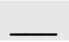 & c & 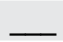 & - & $1 / 2$ & 2 & $x$ & $E$ \\
\hline A. trhiphyllum & castaño oscuro & 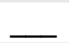 & c & $p$ & ב & $1 / 2$ & 2 & V & $\mathrm{T}$ \\
\hline A. ulbrichtii & verde-castaño & $\mathrm{p}$ & $\begin{array}{l}\text { c (interrumpido } \\
\text { en las alas) }\end{array}$ & $\longrightarrow$ & $\mathrm{p}$ & $1: 8 / 10$ & 2 & $\mathrm{x}$ & $\mathrm{T}$ \\
\hline A. uniseriale & $\begin{array}{l}\text { castaño oscuro } \\
\text { lustroso }\end{array}$ & - & c & $\mathrm{p}$ & 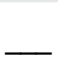 & $1: 2 / 3$ & 2 & $x$ & $\mathrm{~T}$ \\
\hline
\end{tabular}
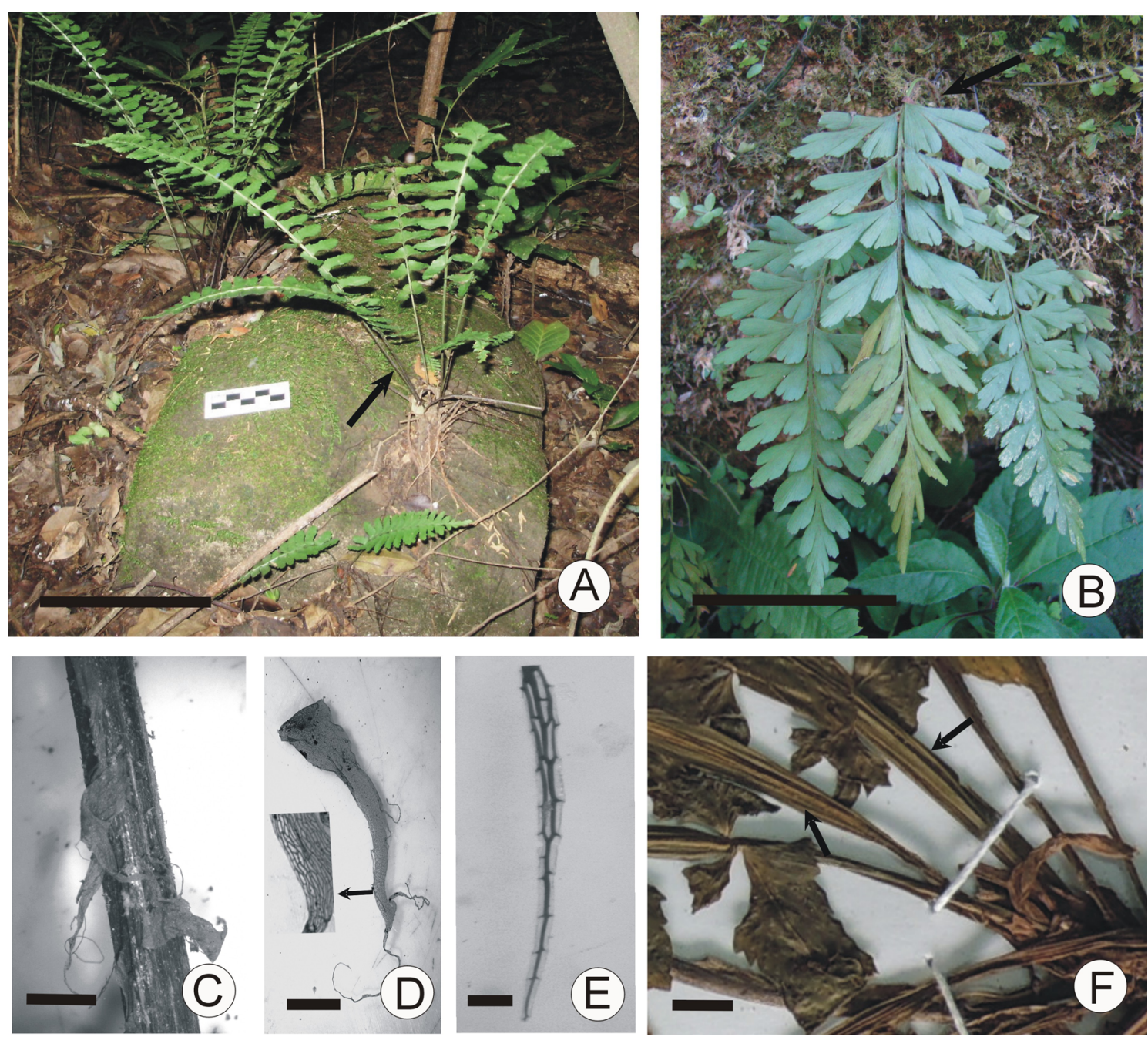

Fig. 1. A-F. Morfología del pecíolo. A: pecíolos oscuros brillosos, erectos (A. argentinum). B: pecíolos verdosos péndulos $(A$. praemorsum). C-E: indumento del pecíolos. C: escamas lanceoladas (flecha) $(A$. squamosum). D: Escama lanceolada (flecha), con margen ciliado y ápice piliforme, clatrada (en detalle) $(A$. squamosum). E: escama filiforme (A. pumilum). F: pecíolos alados (flechas) (A. alatum). Escalas= A-B: 10 $\mathrm{cm}$; C: $1 \mathrm{~mm}$; D-E: $0.25 \mathrm{~mm}$; F: $1 \mathrm{~cm}$. 

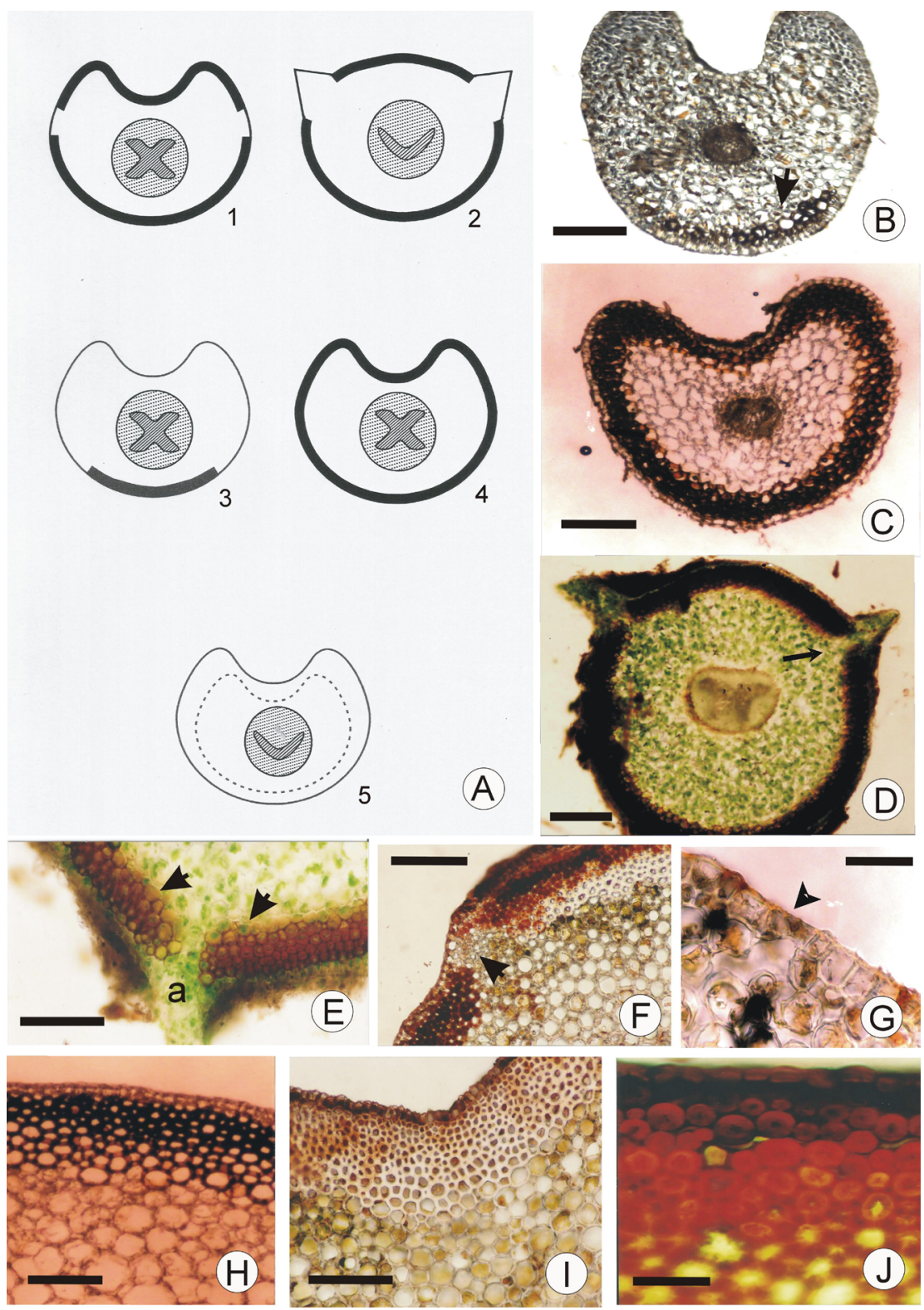

Fig. 2. A-J. Tejido mecánico subepidérmico, líneas de aireación y haces vasculares. A: esquemas de los tipos de ubicación del tejido mecánico (punteado o relleno negro) en sección transversal. 1: tejido mecánico interrumpido en líneas de aireación (punteado). 2: tejido mecánico interrumpido en las alas. 3: tejido mecánico abaxial. 4: tejido mecánico continuo 5: células parenquimáticas con paredes engrosadas (punteado). En 2 y 5 se observa el contorno del xilema en forma de "V"; en 1-3 y 4 en forma de "X". B-J: fotomicrografías con MO. B: CT de pecíolo con tejido mecánico limitado al lado abaxial (flecha) (A. dareoides). C: CT de pecíolo con surco adaxial y tejido mecánico continuo (flecha) (A. uniseriale). D: CT de pecíolo con alas y tejido mecánico interrumpido en las alas (flecha) (A. lorentzii). E: detalle del tejido mecánico interrumpido a nivel de las alas (flechas) (A. ulbrichtii). F: líneas de aireación (flecha) (A. praemorsum). G: estoma (punta de flecha) a la altura de la línea de aireación ( $A$. dareoides). $\mathrm{H}$ : tejido subepidérmico continuo formado por células parenquimáticas con paredes engrosadas ( $A$. scandicinum). I: tejido subepidérmico continuo formado por varias capas de tejido esclerenquimático $(A$. praemorsum). $\mathrm{J}$ : detalle de células del tejido esclerenquimatico $(A$. serra). Escalas=

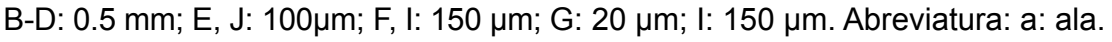


Bol. Soc. Argent. Bot. 54 (2) 2019
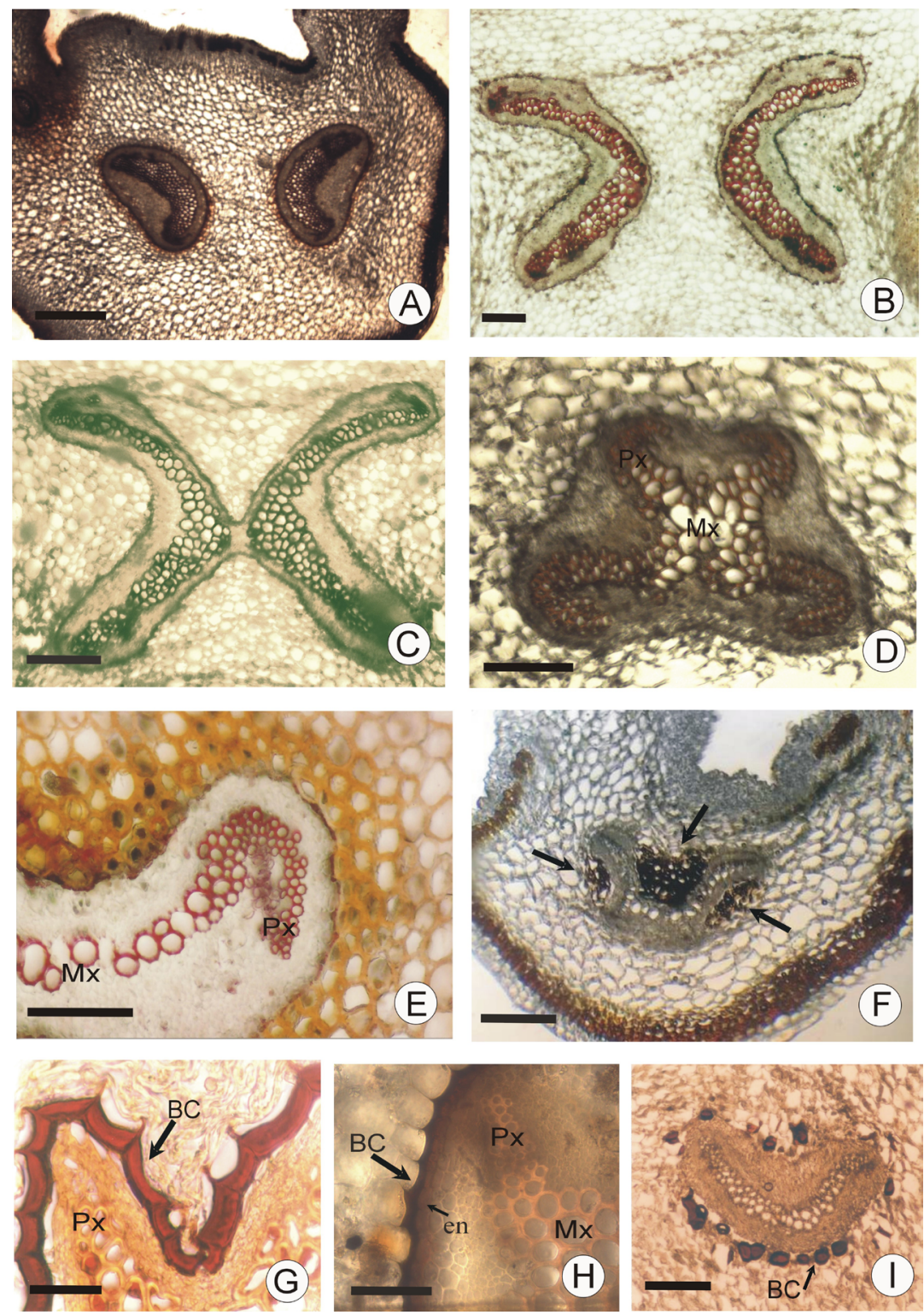

Fig. 3. A-I. Vascularización del pecíolo y banda circumendodermal. A: sección transversal de la base del pecíolo donde se observan dos haces vasculares de contorno ovalado y xilema en forma de "C" (A. squamosum). B: ídem mostrando dos haces vasculares en forma de "C", al igual que el xilema ( $A$. achalense). C: sección transversal de la zona media del pecíolo con un haz vascular en forma de " $X$ " $y$ xilema en forma de "C" (A. achalense). D: ídem con un haz vascular en forma de " $X$ " y xilema en forma de "hipocampo" (A. scandicinum). E: detalle de haz vascular con xilema en forma de "hipocampo" ( $A$. serra). $\mathbf{F}$ : sección transversal de la base del pecíolo con un solo haz en forma de "U" al igual que el xilema, sin banda circumendodermal, con grupos de células esclerenquimatosas alrededor del haz (flechas) $(A$. auritum). G: detalle de la banda circumendodermal conformada por células con paredes tangenciales internas fuertemente engrosadas (flecha) (A. pulchellum). $\mathbf{H}$ : detalle de banda circumendodermal conformada por células con paredes tangenciales internas levemente engrosadas ( $A$. clausenii). I: sección transversal de la zona media del pecíolo con banda circumendodermal interrumpida y haz en forma de " $\mathrm{V}$ " ( $A$. serratum). Escalas $=$ A, B, D: $200 \mu \mathrm{m}$; C: $250 \mu \mathrm{m}$; E, I: $150 \mu \mathrm{m} ; \mathrm{F}: 300 \mu \mathrm{m} ; \mathrm{G}-\mathrm{H}: 100 \mu \mathrm{m}$. Abreviaturas: Mx= metaxilema; $\mathrm{Px}=$ Protoxilema; $\mathrm{BC}=$ banda circumendodermal; en= endodermis. 


\section{Discusión Y CONCLUSIONES}

La morfología de los pecíolos brinda caracteres para la identificación de grupos de especies entre los taxones de Asplenium aquí analizados, resultando diagnósticos el color, la longitud y la presencia/ausencia de alas y su desarrollo. En la clave de identificación de las especies de Asplenium que crecen en Argentina presentada por Ganem et al. (2016), el carácter color del pecíolo permitió diferenciar grupos de especies con lámina 1-pinnada. En cuanto a las alas, este rasgo fue utilizado para segregar especies con láminas enteras o con alto grado de división (3-pinnadas). Particularmente, el grado de desarrollo de las alas permite la identificación de $A$. alatum entre las especies analizadas.

En cuanto a la anatomía del pecíolo, los caracteres que resultan diagnósticos para las especies analizadas son: el tipo de tejido subepidérmico (parenquimático o esclerenquimático) y $\mathrm{su}$ disposición, la presencia o ausencia de la banda circumendodermal y sus características, y el número y contorno de los haces vasculares. El tejido mecánico subepidérmico está ausente en tres de los taxones analizados: $A$. auriculatum, $A$. brasiliense y $A$. gilliesii. En el caso de $A$. auriculatum, nuestras observaciones coinciden con las de Wetzel et al. (2017). En las restantes especies este tejido puede disponerse de forma continua o solo en la zona abaxial, como en A. absscisum, A. auritum, A. balansae, A. inaequilaterale, $A$. mucronatum, cuyos pecíolos son verdosos a castaños, y en $A$. daeroides donde los pecíolos son castaño oscuros.

De acuerdo a Lin \& De Vol (1977b) las líneas de aireación se presentan solo en pecíolos verdes y no alados, pero en este trabajo han sido observadas en pecíolos con otras coloraciones y también en alados, como $A$. achalense, A. alatum y $A$. squamosum, entre otros. Se infiere así que no hay una relación estrecha entre el color del pecíolo, el desarrollo de las alas y las líneas de aireación.

La banda circumendodermal (BC), ubicada en la zona más interna de la corteza de los pecíolos, es característica de varias familias de helechos (Hernández-Hernández et al., 2012). Esta consiste en células con paredes engrosadas no lignificadas (compuestas de celulosa y taninos), con el mismo origen que la endodermis pero sin depósitos de suberina ni lignina. Su función principal es la protección contra agentes patógenos (por contener en sus paredes taninos y fenoles), y como soporte biomecánico, otorgándole flexibilidad al no estar lignificada (Hernández-Hernández et al., 2012). En el presente trabajo, la BC fue observada en varias de las especies analizadas (A. absscisum, A. formosum, A. inaequilaterale, A. kunzeanum, A. lilloanum, A. palmeri, A. pulchellum, A. resiliens, A. sellowianum, A. serratum, A. triphyllum, A. uniseriale), formando un anillo continuo o discontinuo. En aquellos pecíolos sin $\mathrm{BC}$ se registra un tejido mecánico sub-epidérmico esclerenquimático, probablemente cumpliendo la función biomecánica de resistencia, como lo proponen Hernandez-Hernandez et al. (2012). Wetzel et al. (2017) mencionaron que la BC está ausente en A. auriculatum y A. kunzeanum, sin embargo la misma fue observada en este trabajo.

Se ha asociado la presencia de $\mathrm{BC}$ continua con especies con frondes grandes, y la de una $\mathrm{BC}$ discontinua o ausente en aquellas con láminas reducidas, menos divididas (Hernández-Hernández et al., 2012). Sin embargo, en las especies de Asplenium aquí estudiadas, la misma aparece tanto en pecíolos de láminas 1-pinnadas como en aquellas con láminas 3-pinnadas, e igualmente en frondes pequeñas como más desarrolladas.

En casi la totalidad de las especies analizadas se registraron dos haces vasculares en la base de los pecíolos, los cuales se unen en la porción media. Sólo en A. auritum se observó un único haz vascular, con el xilema dispuesto en forma de U, en coincidencia con lo hallado por Wetzel et al. (2017). Khare \& Shankar (1989) encontraron otros patrones de vascularización de los pecíolos, como el de $A$. varians que presenta un solo haz con dos bandas xilemáticas que se unen distalmente, o el de $A$. dalhousiae y A. laciniatum, cuyos pecíolos poseen dos haces que corren libremente sin unirse a lo largo del mismo. Estos patrones no fueron observados en las especies analizadas aquí.

En la porción basal de los pecíolos, las bandas xilemáticas mostraron en general forma de $\mathrm{C} \mathrm{u}$ ovalada, excepto en A. scandicinum, A. serra y A. squamosum, donde estas presentaron forma de hipocampo. Según Ogura (1972) y Lin \& Devol (1977a, b) esta disposición del xilema en los haces no estaría presente en los pecíolos de Asplenium, sin embargo la misma fue reportada previamente por Ganem et al. (2007) para A. pseudonitidum y A. squamosum y por Wetzel et al. (2017) para $A$. 
oligophyllum y corroborada en el presente trabajo, incluyendo su presencia en $A$. scandicinum y $A$. serra.

En la mayoría de las especies analizadas el xilema se une en la porción distal del pecíolo formando una $\mathrm{X}$, excepto en $A$. auriculatum, $A$. gastonis, A. palmeri, A. pumilum, A. serra y $A$. triphyllum donde la configuración del xilema es en forma de $\mathrm{V}$, y en $A$. auritum y $A$. cuspidatum, donde éste se dispone formando una U. Ganem et al. (2007) y Wetzel et al. (2017) reportaron las mismas configuraciones para $A$. achalense, $A$. auriculatum y $A$. squamosum, resultando novedosa la información para el resto de las especies estudiadas en el presente trabajo. Nuestras observaciones en $A$. serra no coinciden con las de Wetzel et al. (2017), quienes mencionan que en este taxón los haces se unen distalmente formando un único haz en forma de $\mathrm{U}$.

La anatomía general del pecíolo no evidenció relación con el hábito de las plantas, coincidiendo de esta manera con lo expresado por HernándezHernández et al. (2012), quienes incluyeron en su estudio distintos grupos de helechos. Así, la BC se observa tanto en especies epífitas como terrestres, coincidiendo con los citados autores.

En general se observa que tanto la $\mathrm{BC}$ como el tejido mecánico subepidérmico están siempre presentes en aquellas especies que viven en ambientes expuestos y más secos. Sin embargo, la $\mathrm{BC}$ se observa también en algunas que crecen en ambientes húmedos, por lo que tampoco se puede relacionar la presencia de la $\mathrm{BC}$ con el ambiente donde habitan las plantas.

De acuerdo a lo aquí observado se pueden encontrar dentro de las Aspleniaceae todos los estados de desarrollo de la BC, desde continua hasta su ausencia total. Estas variaciones podrían reflejar diferentes etapas evolutivas, como lo proponen Hernández-Hernández et al. (2012) para las Thelypteridaceae, con la discontinuidad como etapa intermedia, hacia la pérdida total secundaria de la BC. Futuros estudios filogenéticos moleculares en estas especies permitirían dilucidar esta cuestión.

Dada la elevada cantidad de especies contenidas en Asplenium (unas 700), futuros estudios anatómicos en otros taxones, junto con análisis moleculares, contribuirán a seguir esclareciendo cuestiones sistemáticas en este género.

\section{Contribución de los AUtores}

MAG, MLL y GEG han realizado conjuntamente y a partes iguales la colecta de datos, su interpretación y redacción del manuscrito. OA participó en la escritura del manuscrito.

\section{Agradecimientos}

Los autores agradecen a las Instituciones que aportaron el material y a la UNLP y UNJu por los subsidios recibidos para que este estudio pudiera llevarse a cabo (Proyectos UNLP 11/N610 y 11/ N725; UNJu 08/A115 y 08/A165).

\section{Bibliografía}

D’AMBROGGIO DE ARGÜESO, A.M. 1986. Manual de Técnicas en Histología Vegetal. Ed. Hemisferio Sur, Buenos Aires.

GABANCHO, L.R. \& C. PRADA. 2011. The genus Hymenasplenium (Aspleniaceae) in Cuba, including new combinations for the neotropical species. Amer. Fern J. 101: 265-281. https://doi.org/10.1640/0002-8444-101.4.265

GANEM, M.A., G.E. GIUDICE, M.L. LUNA \& E.R. DE LA SOTA. 2007. Revisión del grupo Asplenium squamosum en América. Candollea 62: 149-156.

GANEM, M.A., M.D. ARANA, M.L. LUNA, O. AHUMADA \& G.E. GIUDICE. 2014. Primer registro de Asplenium harpeodes (Aspleniaceae) para la flora Argentina. Darwiniana, nueva serie 2: 237-242. https://doi.org/10.14522/darwiniana/2014.22.602

GANEM, M.A., G. E GIUDICE \& M. L. Luna. 2016. Asplenium L. In: ZULOAGA, F \& M. BELGRANO (eds.). PONCE, M. M. \& M. ARANA (coords.), Flora Vascular de la República Argentina. Vol. 2. Licofitas, Helechos, Gymnospermae, pp. 54-76. Estudio Sigma, Buenos Aires.

GIUDICE, G. E., M.A. MORBELLI \& M. R. PIÑEIRO. 2002. Palynological study in Aspleniaceae from North-west Argentina. Bol. Soc. Argent. Bot. 37: 217- 229.

GIUDICE G.E., M.L. LUNA \& M.A. GANEM. 2013. El género Hymenasplenium (Aspleniaceae) en Argentina. Darwiniana nueva serie 1: 46-53.

HERNÁNDEZ-HERNÁNDEZ, V., T. TERRAZAS, K. MEHLTRETER \& G. ANGELES. 2012. Studies of petiolar anatomy in ferns: structural diversity and systematic significance of the circuendodermal band. Bot. J. Linn. Soc. 169: 596-610. https://doi.org/10.1111/j.1095-8339.2012.01236.x 


\section{María A. Ganem et al. - Morfología y anatomía de pecíolos de Asplenium}

KATO, M. 1972. The vascular structure and its taxonomic significance in the Athyriaceae. Acta Phytotax. Geobot. 25: 79-91.

KEATING, G. O. 1968. Trends of specialization in the stipe anatomy of Dennstaedtia and related genera. Amer. Fern J. 58: 76-140. https://doi.org/10.2307/1546552

KHARE, P. K. \& R. SHANKAR. 1989. On the petiolar structure of some Asplenium species. Can. J. Bot. 67 (1): 95-103. https://doi.org/10.1139/b89-014

LIN, B. L. \& C.E. DE VOL. 1977a. The use of stipe characters in ferns taxonomy. I. Taiwania 22: 91-99.

LIN, B. L. \& C.E. DE VOL. 1977b. The use of stipe characters in ferns taxonomy. II. Taiwania 23: 88-95.

OGURA, Y. 1972. Comparative anatomy of vegetative organs of the Pteridophytes. In: Zimmermann, W. \& P. G. Ozenda (eds.). Handbuch der pflanzenanatomie, Berlin, Gebrüder Borntraeger.

PONCE, M. M. 1996. Pteridophyta. In: ZULOAGA, F. O. \& O. MORRONE (eds), Catálogo de las plantas vasculares de la República Argentina,
Parte I. Monographs of Systematic Botany, Missouri Botanical Garden 60: 1-79.

SCHNEIDER, H. 1997. Root anatomy of Aspleniaceae and the implications for systematics of this fern family. Fern Gaz. 15: 160-168.

SMITH, A. R. 1981. Asplenium. In: BREEDLOVE, D. E. Flora of Chiapas. Part 2: Pteridophyta. pp. 35-58.

TRYON, R. M. \& R. G. STOLZE. 1993. Pteridophyta of Perú. Part IV: Aspleniaceae-Polypodiaceae. Fieldiana (Bot.) 32: 1-190.

UMIKALSOM, Y. 1992. Anatomical studies of the Malasyan Aspleniaceae and Athyriaceae. Bot. J. Linn. Soc. 110: 111-119. https://doi.org/10.1111/j.1095-8339.1992.tb00285.x

WETZEL, M. L. R, L. D. S. SYLVESTRE, C. F. BARROS \& R. C.VIEIRA. 2017. Vegetative Anatomy of Aspleniaceae Newman from Brazilian Atlantic rainforest and its application in taxonomy. Flora 233: 118-126. https://doi.org/10.1016/j.flora.2017.05.010

ZARLAVSKY, G. E. 2014. Histología Vegetal. Técnicas simples y complejas. pp 1-198 Soc. Argentina de Botánica, Buenos Aires. 
